\title{
Disc-magnetosphere interactions in cataclysmic variable stars
}

\author{
Coel Hellier \\ Astrophysics Group, Keele University, Staffordshire ST5 5BG, U.K. \\ email: ch@astro.keele.ac.uk
}

\begin{abstract}
I review, from an observational perspective, the interactions of accretion discs with magnetic fields in cataclysmic variable stars. I start with systems where the accretion flows via a stream, and discuss the circumstances in which the stream forms into an accretion disc, pointing to stars which are close to this transition. I then turn to disc-fed systems and discuss what we know about how material threads on to field lines, as deduced from the pattern of accretion footprints on the white dwarf. I discuss whether distortions of the field lines are related to accretion torques and the changing spin periods of the white dwarfs. I also review the effect on the disc-magnetosphere interaction of disc-instability outbursts. Lastly, I discuss the temporary, dynamo-driven magnetospheres thought to occur in dwarf-nova outbursts, and whether slow-moving waves are excited at the inner edges of the disc.
\end{abstract}

Keywords. Accretion, accretion disks, magnetic fields, binaries: close, novae, cataclysmic variables, X-rays: stars.

\section{Introduction}

Given that interactions of an accretion disc with a magnetosphere are widespread in astrophysics, one can ask why it is of particular interest to study those in close binary systems such as the cataclysmic variable stars. One answer is that these systems often show a great range of observational clues. Periodic and quasi-periodic behaviour, often on timescales that are easy to study, is the speciality of cataclysmic variables, making them prime systems for advancing our understanding of accretion.

If you want to see how an emission line varies with the spin-cycle of a magnetic white dwarf in a CV, repeated 10 times for reliability, you need only watch for three hours, and the fossil field of the white dwarf will not have changed. In contrast, the same task for a YSO would take weeks, and the dynamo-driven field might be changing over that time. Further, the field of the white dwarf is more likely to be a simple dipole, and thus easier to model.

In this review I make an observationally led overview of the disc-field interactions in cataclysmic variables. I consider, first, the nature of an accretion flow in the presence of a magnetic field, and whether a disc forms, and then turn to how the disc interacts with the field. For a theoretical account of these topics see Li (1999) or Frank, King \& Raine (2002). The definitive review of cataclysmic variable stars is Warner (1995), while for a shorter introduction see Hellier (2001).

One big difference from the situation in YSOs is that in close binaries the accretion originates in a stream from the secondary star through the inner Lagrangian point $\left(L_{1}\right)$. In cataclysmic variables with a highly magnetic white dwarf (exceeding $\sim 30 \mathrm{MG}$ ) the white dwarf is phase-locked to the orbit. In such stars (called AM Her stars or polars) the ballistic stream becomes magnetically controlled and is channelled onto a magnetic pole of the white dwarf. Since this situation is least similar to YSOs I will not deal with 
it here, though for accounts see Wickramasinghe \& Ferrario (2000) and Schwope et al. (2004).

In a handful of AM Her stars the white dwarf is slightly asynchronous with the orbit, despite a high magnetic field. Perhaps these stars have been knocked out of synchronism by a recent nova eruption (e.g. Schmidt \& Stockman 1991). The trajectory of the stream will now change on the 'beat' cycle between the spin and orbital cycles, as the relative orientation of the dipole changes. Typically the accretion stream flips from one pole to the other and back on the beat cycle (10-50 days), though at any one time the star will look like an AM Her with magnetically channelled stream accretion. For accounts of such stars see Ramsay et al. (2000), Ramsay \& Cropper (2002), Staubert et al. (2003) and Schwarz et al. (2005).

The main focus of this review will be the 'intermediate polars', which have weaker fields of 1-10 MG. In this regime the field is usually not strong enough to prevent an accretion disc from forming, but is strong enough to carve out the inner disc and for the magnetosphere to dominate the observed characteristics. The hallmark of IPs is X-ray flux from magnetically channelled accretion onto the white dwarf, heavily pulsed at the white-dwarf spin period. In some systems we also see pulsed polarised light, though in many IPs the polarised light is too diluted by other parts of the system to be detected. For an excellent introduction to these stars see Patterson (1994).

\section{Disc formation: the case of V2400 Oph}

Under what conditions does a disc form around a magnetic white dwarf? If the closest approach of a ballistic stream from the Lagrangian point is further out than the magnetic disruption radius, $R_{\mathrm{mag}}$ (at which the magnetic pressure exceeds the ram pressure of the material), then the stream material will accumulate at the circularisation radius (where the angular momentum of the orbit matches that of the $L_{1}$ point), and spread inwards and outwards until the inner edge meets $R_{\text {mag. }}$. However, while this condition applies in some wide binaries such as GK Per, it doesn't hold for the field strengths and orbital periods typical of most IPs. Thus it appears that most IPs form discs even though the trajectory of a ballistic stream would enter the magnetosphere. The case of V2400 Oph gives clues as to how this might occur.

V2400 Oph is the most plausible candidate for an IP which has no disc. We know the white-dwarf spin period from a clear detection of a 927-s pulsation in polarised light (Buckley et al. 1997), yet there is no X-ray pulse at this period. Instead the X-rays are pulsed at the 1003-s beat period between the spin and orbital cycles (Fig. 1), a clear indication that the accretion proceeds through a stream which flips between the two magnetic poles as their relative orientation changes. Indeed, an X-ray beat pulse had previously been proposed as the main diagnostic of stream-fed accretion in an IP (Hellier 1991; Wynn \& King 1992).

Analysis of how V2400 Oph's spectral lines change with the spin and beat cycles (Hellier \& Beardmore 2002) showed a reasonably good match to a simple model of a pole-flipping stream. And yet the low amplitudes of the pulsations and the absence of any orbital modulation indicates that this cannot be the whole story. Hellier \& Beardmore (2002) argued for the presence of additional orbiting material that is diluting the emission from the stream. Yet this cannot be an accretion disc, since our experience of these stars suggests that this would produce an X-ray spin-period pulsation.

Instead, we turn to the 'diamagnetic blob' scenario developed by Wynn \& King (1995), which suggests that dense 'blobs' of material can orbit quasi-ballistically, but with the addition of a 'magnetic drag' as they cross field lines. Blobs originating in the accretion 


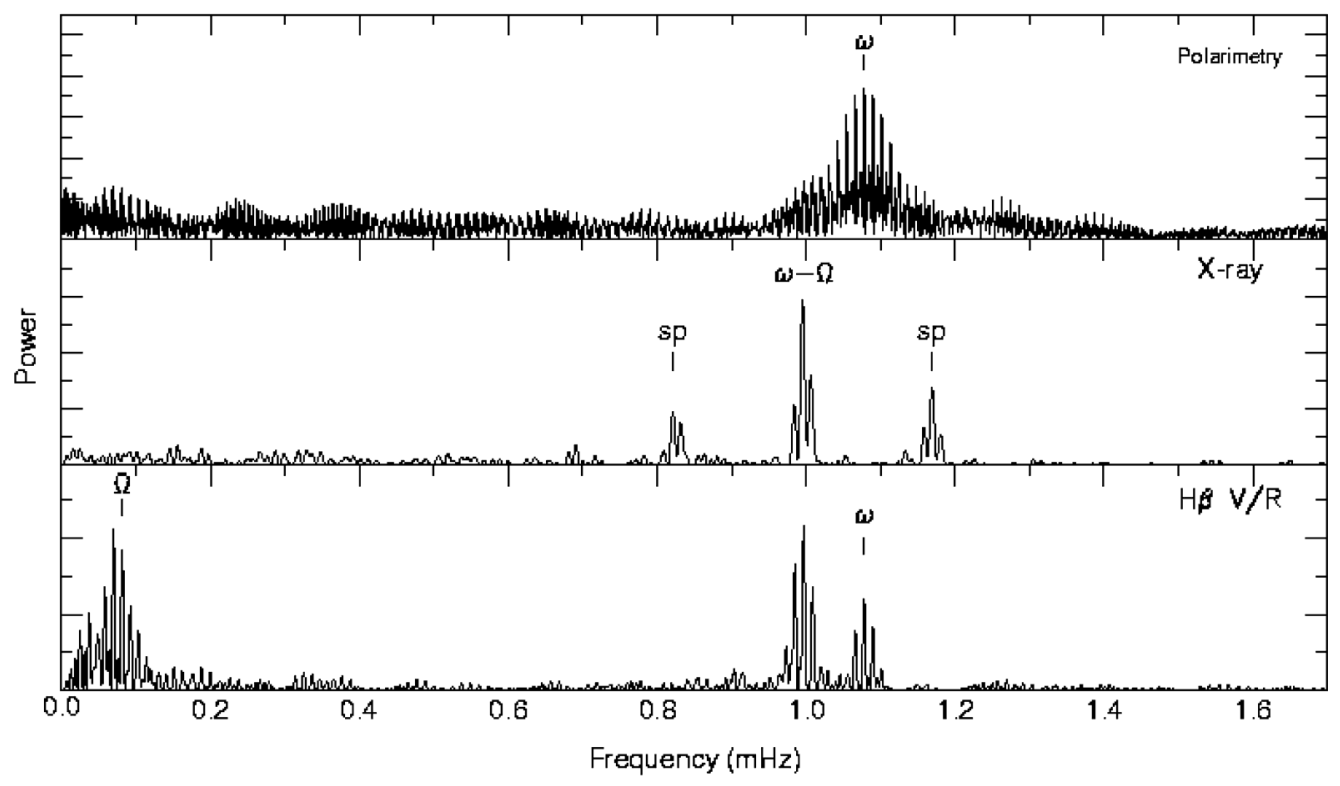

Figure 1. The Fourier transforms of optical polarimetry, X-ray flux and emission line radial velocity for V2400 Oph. The polarimetry reveals the 927-s spin period (denoted $\omega$ ) while the $\mathrm{X}$-ray flux varies instead at the beat period $(\omega-\Omega$, where $\Omega$ is the orbital frequency). The emission-line radial velocities vary with all three periods. Figure from Hellier (2002), using data by Hellier and David Buckley.

stream could orbit, lose energy as they cross field lines, spiral inwards and accrete. Yet they would also tend to screen the field from each other. The balance of the two factors would determine whether the blobs accumulate sufficiently to form a disc, and it appears that V2400 Oph is close to this borderline, with sufficient orbiting blobs to carry much of the accretion flow (thus diluting the stream-fed pulsations), yet with the blobs not 'organised' enough to form a disc or to produce an X-ray spin pulse.

V2400 Oph has a field strength (estimated as 9-27 MG; Buckley et al. 1995; Väth 1997) that is as high or higher than in any other IP, and this may explain why the orbiting blobs cannot quite form a disc in this star, whereas they do in most IPs.

A possibly similar case is that of TX Col. Again, the main diagnostic of stream-fed versus disc-fed accretion is the ratio of the beat-cycle to the spin-cycle pulsations in the X-ray lightcurve. In TX Col the two pulsations are of comparable magnitude, with the spin pulse being larger at some epochs and the beat pulse at others (Norton et al. 1997; Wheatley 1999). So it appears that in TX Col the orbiting material has managed to organise into some sort of disc, yet just as much of the flow is still in the form of a stream flipping between the poles, and the relative proportions of the two fluctuate with time. Interestingly, Mhlahlo et al. (2007b) report the detection of high-amplitude quasiperiodic oscillations in the optical light of TX Col, at a period of $6000 \mathrm{~s}$ that is unrelated to the spin or beat periods. Understanding these pulsations could be an important clue to an accretion flow on the verge of disc formation.

\section{EX Hya-like IPs}

For the magnetosphere to be spinning in equilibrium with a disc (meaning that the corotation velocity is close to the Keplerian velocity at the inner disc edge) the spin 
period cannot exceed $0.1 P_{\text {orb }}$ (e.g. King \& Lasota 1991). This arises since the inner disc edge cannot be further out than the circularisation radius, otherwise the disc would lose more angular at its inner edge than it gains from the stream, and it couldn't survive.

At least three stars, EX Hya, V1025 Cen and DW Cnc, clearly exceed this limit, and are instead in a state where the corotation radius is near the Lagrangian point. One possibility is that they are spinning much more slowly than equilibrium, perhaps as a result of having previously been in a discless state (and it is worth noting that EX Hya has been spinning up monotonically for as long as we've been observing it; e.g. Hellier \& Sproats 1992).

The alternative possibility is that these stars currently have no disc. Although there is not yet a consensus on this (e.g. Hellier et al. 1987; Hellier, Wynn \& Buckley, 2002; Belle et al. 2005; Mhlahlo et al. 2007a) my own view is that they probably do have discs. The main reasons are (1) neither EX Hya nor V1025 Cen shows an X-ray beat pulse in its usual quiescent state, implying that the accretion loses all memory of orbital phase, and (2) neither star shows polarisation, whereas the discless idea requires them to be among the highest-field IPs, with magnetic fields dominating most of the way to $L_{1}$. Further, EX Hya shows outbursts during which the accretion stream does overflow the disc and go as far as the magnetosphere; the signs of this are obvious, including an X-ray beat-cycle pulsation, eclipse profiles of a stream, and high-velocity features in the emission lines (e.g. Hellier et al. 2000), which increases our confidence that accretion is purely disc-fed in quiescence when these features are not seen. $\dagger$

Why do these three stars with longer-than-expected spin periods cluster at short periods? One explanation might be that in smaller, shorter-period binaries the synchronisation torques (thought to be caused by interaction of the magnetic fields of the primary and secondary) are stronger. Thus, if a low state of no mass transfer leads the disc to dissipate, a shorter-period system might relatively quickly head for synchronism whereas a longer-period system would not. Once mass-transfer and a disc are re-established the systems would spin back up, but they would still have a higher probability of being found with long, non-equilibrium spin periods.

\section{The disc-fed IPs}

In Fig. 2 I plot the spin and orbital periods of the known IPs. I have been conservative in choosing what to include, placing emphasis on detections of a periodicity in multiple data sets, preferably including X-ray data.

The line at $P_{\text {spin }}=0.1 P_{\text {orb }}$ indicates the expected location for equilibrium rotation of a discless accretor, such as V2400 Oph. Systems above this line cannot both possess discs and be in equilibrium. Other than the short-period systems just discussed, most IPs sit on or below this line, compatible with disc-fed accretion. Further interpretation of the $P_{\text {spin }} / P_{\text {orb }}$ value is hard without knowledge of the magnetic moment or the radius of the inner disc edge, neither of which we have in most IPs. Norton, Wynn \& Somerscales (2004) attempt to extract such information from the $P_{\text {spin }} / P_{\text {orb }}$ values, but their method

$\dagger$ Mhlahlo et al. argue that the magnetic influence does go as far as the outer edge of the disc in EX Hya in quiescence. The main argument for this is the detection of emission with the low velocity typical of the outer disc which nevertheless appears to circle with the spin cycle. However, EX Hya is peculiar in having a spin period very close to $2 / 3 \mathrm{rds}$ of the orbit; thus orbital-cycle variations do not smear out when folded on the spin cycle (see Hellier et al. 1987). The feature seen by Mhlahlo et al. is simply the usual orbital S-wave from the edge of the disc, which contaminates the plots of data folded on the spin cycle. 


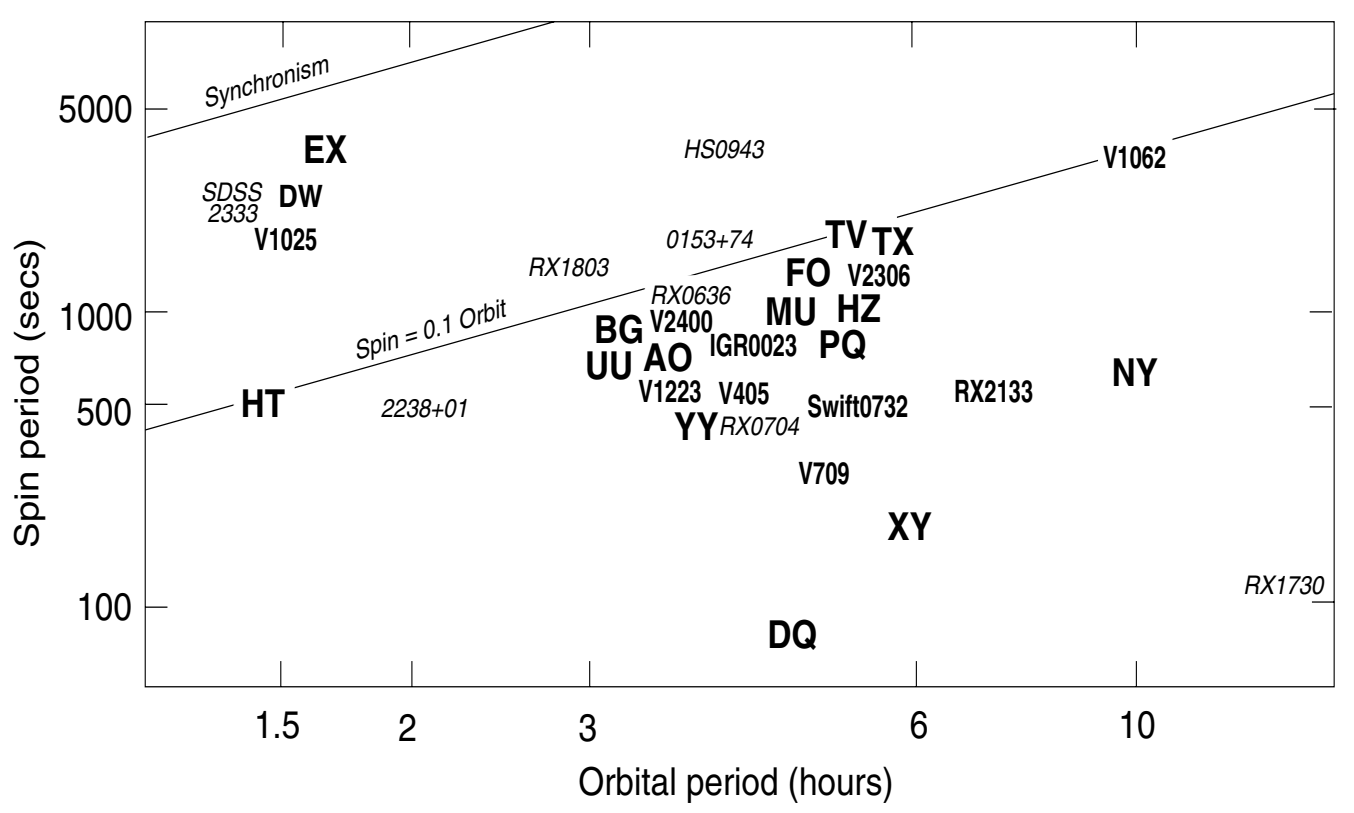

Figure 2. The spin and orbital periods of the intermediate polars, indicated by abbreviated names (some labels have been moved marginally for clarity). Italics indicate that the location on the plot is uncertain, or that the system is not fully secure as an IP, perhaps lacking confirmation from multiple datasets or authors. For details of each system see the compilation by Koji Mukai at the 'IP home page' http://lheawww.gsfc.nasa.gov/users/mukai/iphome/iphome.html

is predicated on a magnetically dominated, non-disc accretion flow, and so would not be applicable to systems with discs.

Observationally, the evidence is that most of these systems do have discs, which is deduced primarily from the fact that the dominant X-ray pulsation is at the spin period (though see Hellier 1991 for a discussion of other indicators). It is also worth noting that none of the IPs appear to show the 'soft X-ray excess' that is sometimes seen in AM Her stars (see Ramsay \& Cropper 2004 for AM Her stars and Evans \& Hellier 2007 for IPs). The interpretation of soft excesses in AM Hers is that blobbiness of the accretion flow can survive as far as the white-dwarf surface, and that such blobs would not undergo a hard-X-ray-emitting shock but would penetrate the white-dwarf surface and thermalise to produce soft-X-ray emission (e.g. Kuijpers \& Pringle 1982). The absence of this effect in IPs suggests that any such blobs are destroyed, being shredded in an accretion disc, or at least during multiple orbits of the white dwarf.

\subsection{Stream-overflow in disc-fed stars}

Possession of an accretion disc does not guarantee that all the accretion flows through it. As pointed out by theorists (e.g. Lubow 1989) the scale height of the stream from the $L_{1}$ point is likely to exceed that of the outer disc edge, such that part of the flow continues quasi-ballistically. Around the same time, observers were seeing direct indications of this (e.g. Hellier et al. 1989). In many cataclysmic variables the stream will continue inward to some extent, eventually being subsumed into the disc, but if the accretion stream flows as far as the magnetosphere it will produce a beat-cycle X-ray pulsation. The relative power in the beat- and spin-cycle pulsations then presumably gives an indication of the fractions of material accreting by the two paths. 
FO Aqr, AO Psc, BG CMi and V1223 Sgr are all IPs that have a dominant X-ray modulation at the spin period, but which show a weaker, intermittent pulsation at the beat period, presumably resulting from an overflowing stream (e.g. Hellier 1993; 1998; Norton, Beardmore \& Taylor 1996; Beardmore et al. 1998).

In addition, observations of the UV lines of AO Psc with HST have enabled a more direct observation of the interaction of the overflowing stream with the field. At orbital phases when the stream is in front of the strong UV backlight of the white dwarf, we see narrow absorption dips, presumably from stream material (Hellier \& van Zyl 2005). These dips show rapid velocity changes related to the spin cycle, which reveal the stream flailing around in accordance with the orientation of the magnetic field.

\subsection{Spin-period changes}

In addition to spin periods we can ask about spin-period changes [see Mukai (2007) for a compilation of data]. Long term monitoring of IPs has found systems where the white dwarf is spinning up (AO Psc, BG CMi, EX Hya), and systems where it is spinning down (PQ Gem, V1223 Sgr), and at least one system that shows episodes of both (FO Aqr). Of course we have monitored these systems for only a small fraction of evolutionary timescales, so it would be dangerous to overinterpret these results. However, the short timescales of the period changes and the fact that at least one has swapped from spin up to spin down over a decade (FO Aqr; Williams 2003) suggests that a typical IP below the line in Fig. 2 is hovering about an equilibrium spin period.

A decade ago, Patterson (1994) described this issue as "murky" and suggested that a treasure-trove of $\dot{P}$ data awaited anyone with a good enough theory to interpret it. We are still waiting, and few authors have been brave enough to go beyond Patterson's review.

\section{The disc-field connection}

One of the hardest questions is what does the connection between the disc and the field look like? What is the radial extent over which disc material feeds onto field lines? What is the vertical extent? What does the vertical section look like?

We have very few observational clues, but one approach is to use eclipses, where the occulting knife edge of the secondary can give spatial information on the X-ray-emitting accretion regions. By tracing these back along field lines, we can deduce information about the disc-magnetosphere interface.

So far, this technique is only possible in XY Ari, which is the only known IP with deep X-ray eclipses. A study by Hellier (1997b) found that the X-ray eclipse egresses occurred in a short enough time that the accretion footprints covered a linear extent of less than 0.1 white-dwarf radii. This suggests that the feeding onto field lines is restricted to a relatively small azimuthal range. That is, at least in quiescence; see $\S 6$ for the changes wrought by an outburst.

Another, more widely applicable technique is to deduce the shapes of the accretion footprints on the white dwarf by interpreting X-ray spin-pulse profiles.

A useful star is HT Cam, which appears to have a very simple X-ray spin pulse, dependent only on whether or not the accretion footprint is on the visible face of the white dwarf. It shows no phase-varying absorption, being devoid of the prominent absorption dips, produced when the accretion flow passes in front of the white dwarf, that are so characteristic of IPs.

Evans \& Hellier (2005b) showed that one of the simplest possibilities adequately models HT Cam's spin pulse. In the model, which used a centred dipole, the intensity of the 

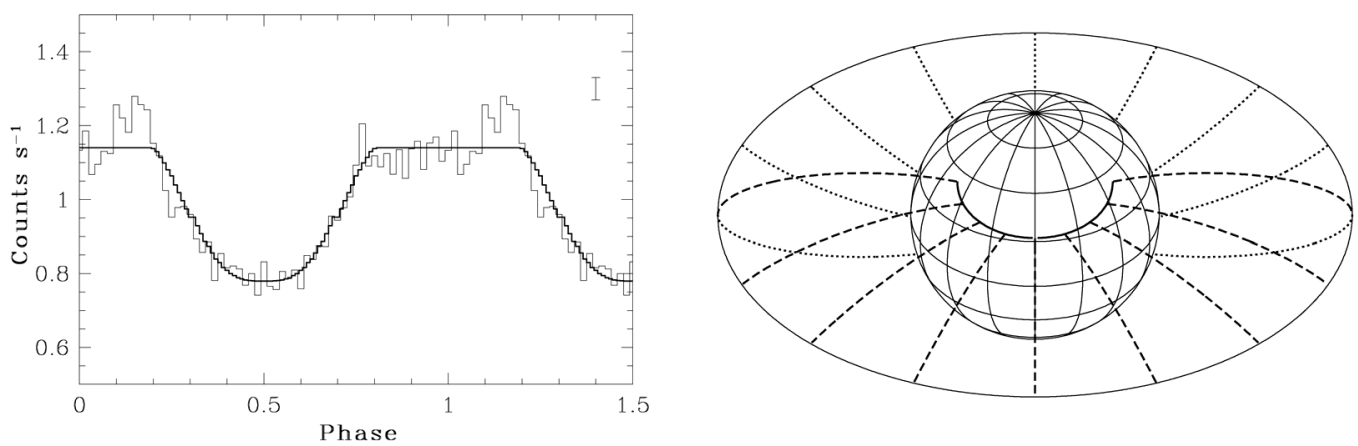

Figure 3. The 'simplest' X-ray spin pulse of HT Cam. The left-hand panel shows the X-ray pulse profile and a fitted model, which is simply a geometric projection of the accretion regions illustrated in the right-hand panel. Figures from Evans \& Hellier (2005b).

footprint as a function of magnetic latitude (which presumably maps to accretion rate as a function of azimuth at the inner edge of the disc) was a $\cos ^{2}$ function which peaked at the azimuth to which the magnetic pole pointed, falling to zero $90^{\circ}$ away, and then doing the same for the other pole.

V405 Aur, however, shows a more complex, double-peaked spin pulse. In order to explain this Evans \& Hellier (2004) suggested that the magnetic dipole was off-centred from the white dwarf, and that the magnetic axis is highly inclined to the spin axis.

All such work is bedevilled by the number of parameters that one could vary in a model (e.g. using multipole, non-symmetric fields) so it should be cautioned that the proposed models may not be unique, with more complex possibilities also possible.

A similar technique to using X-ray pulse profiles is to used spin-pulse profiles of polarised optical light (e.g. Potter et al. 1997; 1998). The idea here is that pulsed polarised light originates near to the white dwarf, and so maps to the accretion footprints, whereas unpolarised optical light, even that pulsed on the spin cycle, likely originates from 'accretion curtains' much bigger than the white dwarf. Where both X-ray and polarimetric data combine we can have some confidence in our interpretations, even in complex cases such as PQ Gem, discussed next.

\subsection{Twisting of the field lines}

The 'simplest' IP spin pulse of HT Cam is compatible with field lines that are undistorted. However, detailed analysis of the complex spin pulse of PQ Gem, using X-ray data, optical spectroscopy, and polarimetry, suggests that the accreting field lines are distorted by accretion torques, and that the accreting field lines are 0.1 cycles ahead of the magnetic pole (Mason 1997; Potter et al. 1997; Hellier 1997a; Evans, Hellier \& Ramsay 2006).

Similar analysis of FO Aqr yields the opposite: the accreting field lines appear to lag the magnetic pole by a quarter of a cycle (Evans et al. 2004).

An obvious question is whether these twists are related to the torques on the white dwarf, as shown by period changes. Indeed, PQ Gem, with field lines swept ahead of the pole, is found to be spinning down (Mason 1997). FO Aqr, with field lines swept behind the pole, is spinning up - at least at some epochs. But it has also shown a period of spin down (Williams 2003); and thorough investigation of the X-ray spin pulse over the different epochs (e.g. Beardmore et al. 1998) show no obvious shifts in the phases of absorption dips that would imply that the field lines had swapped from trailing to leading.

Thus there is no simple interpretation of the current information on field-line twists and period changes; as yet we have such information on too few systems to discern patterns. 


\section{Disc-Magnetosphere interactions in outburst}

We presume that in many of the IPs the white-dwarf magnetospheres are rotating close to equilibrium with their discs. However, cataclysmic variables also show dwarfnova outbursts, where a hydrogen-ionisation instability in the disc causes a hundred-fold increase in the accretion rate, lasting typically for several days (e.g. Lasota 2001; Osaki 2005). In a few cases we have observed outbursts in IPs, which allows us to watch the dynamic behaviour of a magnetosphere.

Theory tells us (e.g. Frank et al. 2002) that as the ram pressure of the accretion flow increases the magnetosphere shrinks (as $r \propto \dot{M}^{-2 / 7}$ ). In an outburst of XY Ari we saw confirmation of this, with major changes in the X-ray pulse profiles indicating that the disc had pushed inwards and blocked the view of the lower magnetic pole (Hellier, Mukai \& Beardmore 1997). It took about a day for the disc to push inwards from $\sim 9 R_{\mathrm{wd}}$ to $\sim 4 R_{\mathrm{wd}}$, and eclipse timings indicate that, when it had done so, the flow of material onto field lines was no longer restricted in azimuthal extent, but now flowed from all parts of the inner disc, to fill a complete ring of magnetic longitude around the magnetic poles. It is unclear whether this change is due more to the non-equilibrium rotation (presumably after the disc has pushed inward the Keplerian velocity at its inner edge would now exceed the speed at which the magnetosphere rotates) or whether it relates to the greater scale height of the disc during outburst.

GK Per has an exceptionally long orbital period, and thus a very large disc with longlived outbursts that are thus easy to study. Analysis of the X-ray pulse profiles suggests that GK Per behaves similarly to XY Ari. The pulse-profiles are explainable by the disc pushing inwards and cutting off the view of the lower pole. Again, the accretion appears to feed from all disc azimuths at the height of outburst, causing complete rings of accretion at the magnetic poles (Hellier, Harmer \& Beardmore 2004; see also Vrielmann, Ness \& Schmidt 2005).

Thus it is clear that observations of IPs in outburst can reveal important observations of the dynamic interaction of a disc and magnetosphere. However, the fact that such outbursts are occasional, short-lived and unpredictable makes such data hard to obtain. In addition to XY Ari and GK Per, YY Dra (Szkody et al. 2002) and HT Cam (Ishioka et al. 2002) are among the few systems where this has been achieved. Some IPs (EX Hya, V1223 Sgr \& TV Col) show even shorter-lived outbursts, lasting only a day, that are even harder to study. So far it is unclear whether these are disc-instability outbursts (shorterlived and lower-amplitude owing to the magnetic truncation of the disc) or whether they are episodes of mass transfer from the secondary star (see, e.g., Hellier et al. 2000 and references therein).

\section{Temporary magnetospheres: DNOs}

A long-studied feature of dwarf-nova outbursts is the occurrence of 'dwarf-nova oscillations' in the lightcurves. These are semi-stable periodicities with periods of 6 to 50 secs, which are presumably related to the white-dwarf spin. The puzzle has been why, if they indicate a spinning magnetosphere, are they seen only in outburst in what are otherwise considered to be non-magnetic systems? Surely the magnetic field should be most manifest in quiescence, when the accretion rate is least? The resolution proposed in the series of papers Warner \& Woudt (2002), Woudt \& Warner (2002), Warner, Woudt \& Pretorius (2003) is that the field is only present in outburst, being generated by a dynamo caused by an equatorial belt which is spun up by the extra accretion in outburst 


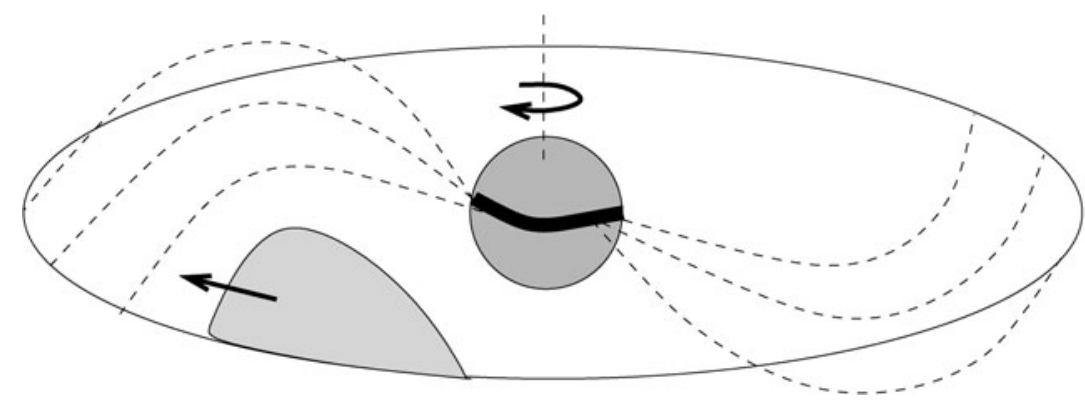

Figure 4. A schematic illustration of a dwarf nova in outburst. While these systems are nonmagnetic is quiescence, the increased accretion of outburst spins up an equatorial belt. This slips over the white dwarf, forming a dynamo and generating a temporary magnetosphere. At the inner edge of the disc slow-moving bulges are produced by prograde travelling waves.

and is slipping over the body of the white dwarf. The low-coherence of the oscillations is then explained by the low inertia of the equatorial belt.

The phenomenology of DNOs is too vast to summarise here (see Warner 2004, Pretorius, Warner \& Woudt 2006 and references therein), but an important topic is their link to the 'quasi-periodic oscillations' sometimes seen in dwarf novae.

\section{QPO waves in the inner disc?}

Quasi-periodic oscillations are longer-period than DNOs and less coherent (for an account of the phenomenology see Warner 2004). Although they have been known for decades, it is only relatively recently that a plausible explanation has been proposed. Warner \& Woudt (2002) suggest that QPOs are caused by travelling waves at the inner edge of the disc, excited by the interplay of the dynamo-generated field with the inner disc. The waves are slow-moving, prograde bulges that modulate the observed light by periodically obscuring the white dwarf as they circle. Empirically, they appear to circle $\approx 15$ times more slowly than the DNO period, which is presumably the rotation period of the temporary, dynamo-driven magnetosphere.

Such bulges can also produce a phenomenon by re-processing the light from the DNO, leading to a quasi-periodicity at the beat period of the DNO and the QPO (see Woudt \& Warner 2002; Marsh \& Horne 1998).

An interesting question is whether these travelling waves are peculiar to the conditions of a dwarf-nova outburst, or whether they are widespread in disc-magnetosphere interactions. Warner et al. (2003) present empirical evidence that the $P_{\mathrm{QPO}}=15 P_{\mathrm{DNO}}$ relation holds in a wide range of objects, from neutron stars to black holes. We can thus ask whether the phenomenon is also present in IPs, with their permanent, fossil fields.

One IP almost certainly showing this phenomenon is GK Per, which in outburst shows large-amplitude absorption dips, recurring quasi-periodically with a 5000-s timescale, 15 times longer than the 351-s spin period (Watson, King \& Osborne 1985).

One proposed model (e.g. Morales-Rueda, Still \& Roche 1999) suggests that the 5000-s timescale is the beat period between the 351-s spin period and the Keplerian velocity at the inner disc edge. However Hellier \& Livio (1994) show that, phenomenologically, the modulation must be caused by periodic obscuration by structure circling at $5000 \mathrm{~s}$. Such structure is then best explained by the Warner \& Woudt (2002) idea.

Are the travelling-wave bulges there only in the non-equilibrium conditions of outburst, or are they present also in quiescence? It is hard to tell. As discussed in Hellier, Harmer 
\& Beardmore (2004), the inner disc edge will be $\sim 5$ times further out in quiescence, meaning that, at the inclination of GK Per, the bulges would not obscure the white dwarf and create absorption dips.

Thus, it may be that we see the QPOs readily enough in dwarf novae, where the weak, dynamo-driven magnetospheres place the bulges near to the white dwarf, where obscuration effects will be easily seen. But in IPs, with stronger fields and larger magnetospheres, the inner disc edges are too far from the white dwarf for obscuration by bulges to be obvious.

It is also worth noting that in most IPs a quasi-periodicity at 15 times the spin period would be close to the orbital cycle, and thus hard to distinguish from orbital-cycle modulations, which are often prominent (e.g. Hellier, Garlick \& Mason 1993; Parker, Norton \& Mukai 2005).

Also, given that QPO effects are usually subtle, they would require trains of data of 10 cycles or more to detect, and this is a timescale $(30 \mathrm{hrs})$ on which it is very hard to obtain continuous data trains. Thus for practical reasons, the question of how widespread this phenomenon is in IPs is very hard to answer.

\section{Concluding remarks}

I have shown above that in many cases observations of magnetic cataclysmic variables give robust clues to how an accretion disc interacts with a magnetosphere. For completeness, I briefly mention two further scenarios. First, there is the possibility of a magnetosphere spinning sufficiently fast to expel material, forming a 'propeller' system. AE Aqr is thought to be in this state, with expulsion of material powered by a rapid spin-down of the white dwarf (e.g. Wynn, King \& Horne 1997; Meintjes \& de Jager 2000; Meintjes \& Venter 2005).

Another possibility is that of a white dwarf with a misaligned spin axis, which is precessing. This would introduce a further periodicity at the precession period into the panoply of cataclysmic-variable periodicities. This has been invoked for stars such as FS Aur and HS 2331+3905 that show periodicities which are otherwise very hard to explain (e.g. Tovmassian et al. 2003; Araujo-Betancor et al. 2005; Tovmassian, Zharikov \& Neustroev 2007).

One notable feature, however, is that owing to the diversity of phenomena even within the intermediate polars, we are often interpreting observational features seen in only one or two systems, rather than analysing patterns seen throughout the class.

There is only one propeller system (AE Aqr); FS Aur and HS 2331+3905 are both unique; only one system (V2400 Oph) is clearly discless; only two systems (EX Hya and GK Per) are well studied in outburst, and both are very different from each other. We have only one system with deep X-ray eclipses (XY Ari, hidden behind a molecular cloud where it cannot be studied in the optical) and one grazing eclipser (EX Hya). Studies of accretion-curtain twists and their relation to spin-period changes are available for only a couple of systems (FO Aqr and PQ Gem); studies of the formation mechanism of the X-ray spin pulses are available for half a dozen stars, but they show a wide range of behaviours, some being single pulsed at the white-dwarf spin period and others being double pulsed (e.g. Evans \& Hellier 2005a).

Thus, progress in understanding disc-magnetosphere interactions in these stars is likely to come from studying sufficient systems in detail to look for patterns encompassing the class. Assisting this is the fact that, in the past 5 years, fully a dozen objects have been added to the number we can plot on Fig. 2. Most of these are only lightly studied, and 
thus the prospects for learning much more about disc-magnetosphere interactions from this class of objects are bright.

\section{References}

Araujo-Betancor, S., et al. 2005, A\& A, 430, 629

Beardmore, A.P., Mukai, K., Norton, A.J., Osborne, J.P., \& Hellier, C. 1998, MNRAS, 297, 337

Belle, K.E., Howell, S.B., Mukai, K., Szkody, P., Nishikida, K., Ciardi, D.R., Fried, R.E., \& Oliver, J.P. 2005, AJ, 129, 1985

Buckley, D.A.H., Sekiguchi, K., Motch, C., O’Donoghue, D., Chen, A-L., Schwarzenberg-Czerny, A., Pietsch, W., \& Harrop-Allin, M.K., 1995, MNRAS, 275, 1028

Buckley, D.A.H., Haberl, F., Motch, C., Pollard, K., Schwarzenberg-Czerny, A., \& Sekiguchi, K. 1997, MNRAS, 287, 117

Evans, P.A., \& Hellier, C. 2004, MNRAS, 353, 447

Evans, P.A. \& Hellier, C. 2005a, in J.-M. Hameury, J.-P. Lasota (eds), The Astrophysics of Cataclysmic Variables and Related Objects, ASP Conf. Proc., 330, 165.

Evans, P.A., \& Hellier, C. 2005b, MNRAS, 359, 1531

Evans, P.A., \& Hellier, C. 2007, ApJ, 663, 1277

Evans, P.A., Hellier, C., \& Ramsay, G. 2006, MNRAS, 369, 1229

Evans, P.A., Hellier, C., Ramsay, G., \& Cropper, M. 2004, MNRAS, 349, 715

Frank, J., King, A.R., \& Raine, D. 2002, Accretion Power in Astrophysics, (Cambridge: CUP)

Hellier, C. 1991, MNRAS, 251, 693

Hellier, C. 1993, MNRAS, 265, L35

Hellier, C. 1997a, MNRAS, 288, 817

Hellier, C. 1997b, MNRAS, 291, 71

Hellier, C. 1998, Adv. Sp. Res., 22, 973

Hellier, C. 2001, Cataclysmic Variable Stars (Springer-Praxis: Chichester)

Hellier, C. 2002, in B.T. Gänsicke, K. Beuermann, K. Reinsch (eds), The Physics of Cataclysmic Variables and Related Objects, ASP Conf. Proc., 261, 92.

Hellier, C., \& Beardmore, A.P. 2002, MNRAS, 331, 407

Hellier, C., Garlick, M.A., \& Mason, K.O. 1993, MNRAS, 260, 299

Hellier, C., Harmer, S., \& Beardmore, A. P. 2004, MNRAS, 349, 710

Hellier, C., Kemp, J., Naylor, T., Bateson, F.M., Jones, A., Overbeek, D., Stubbings, R., \& Mukai, K. 2000, MNRAS, 313, 703

Hellier, C., \& Livio, M. 1994, ApJ, 424, L57

Hellier, C., Mason, K.O., Rosen, S.R., \& Cordova, F.A., 1987, MNRAS, 228, 463

Hellier, C., Mason, K.O., Smale, A.P., Corbet, R.H.D., O’Donoghue, D., Barrett, P.E., \& Warner, B., 1989, MNRAS, 238, 1107

Hellier, C., Mukai, K., \& Beardmore, A. P. 1997, MNRAS, 292, 397

Hellier, C., \& Sproats, L.N. 1992, IBVS, 3724

Hellier, C., Wynn, G.A., \& Buckley, D.A.H. 2002, MNRAS, 333, 84

Hellier, C., \& van Zyl, L. 2005, ApJ, 626, 1028

Ishioka, R., et al. 2002, PASJ, 54, 581

King, A.R., \& Lasota, J.-P. 1991, ApJ, 378, 674

Kuijpers, J.; \& Pringle, J. E., 1982, A\& $A$, 114, L4

Lasota, J.-P. 2001, New Astron. Revs, 45, 449

Li, J. 1999, in C. Hellier, K. Mukai (eds.), Annapolis Workshop on Magnetic Cataclysmic Variables, ASP Conf. Ser., 157, 235

Lubow, S.H., 1989, ApJ, 340, 1064

Marsh, T.R., \& Horne, K. 1998, MNRAS, 299, 921

Mason, K.O. 1997, MNRAS, 285, 493

Meintjes, P. J., \& Venter, L. A. 2005, MNRAS, 360, 573

Meintjes, P. J., \& de Jager, O. C. 2000, MNRAS, 311, 611

Mhlahlo, N., Buckley, D.A.H., Dhillon, V.S., Potter, S.B., Warner, B., \& Woudt, P.A. 2007a, MNRAS, 378, 211 
Mhlahlo, N., Buckley, D.A.H., Dhillon, V.S., Potter, S.B., Warner, B., Woudt, P., Bolt, G., McCormick, J., Rea, R., Sullivan, D.J.. \& Velhuis, F. 2007b, MNRAS, in press (arXiv0705.3259)

Morales-Rueda, L., Still, M.D., \& Roche, P., 1999, MNRAS, 306, 753

Mukai, K. 2007, IP home page, http://lheawww.gsfc.nasa.gov/users/mukai/iphome/iphome.html

Norton, A.J., Beardmore, A.P., \& Taylor, P. 1996, MNRAS, 280, 937

Norton, A.J., Hellier, C., Beardmore, A.P., Wheatley, P.J., Osborne, J.P., \& Taylor, P. 1997, MNRAS, 289, 362

Norton, A.J., Wynn, G.A., \& Somerscales, R.V. 2004, ApJ, 614, 349

Osaki, Y. 2005, Proc. Japan Acad., Ser. B: Physical and Biological Sciences, 81, 291

Parker, T.L., Norton, A.J., \& Mukai, K. 2005, A\&广A, 439, 213

Patterson, J. 1994, PASP, 106, 209

Potter, S.B., Hakala, P.J., \& Cropper, M. 1998, MNRAS, 297, 1261

Potter, S.B., Cropper, M., Mason K.O., Hough, J.H., \& Bailey, J.A. 1997, MNRAS, 285, 82

Pretorius, M.L., Warner, B., \& Woudt, P.A. 2006, MNRAS, 368, 361

Ramsay, G., \& Cropper, M. 2002, MNRAS, 334, 805

Ramsay, G., \& Cropper, M. 2004, MNRAS, 347, 497

Ramsay, G., Potter, S., Cropper, M., Buckley, D.A.H., \& Harrop-Allin, M.K. 2000, MNRAS, 316,225

Schmidt, G.D., \& Stockman, H.S. 1991, ApJ, 371, 749

Schwarz, R., Schwope, A.D., Staude, A., \& Remillard, R.A. 2005, A\&A, 444, 213

Schwope, A. et al. 2004, in S. Vrielmann, M. Cropper (eds), Magnetic Cataclysmic Variables, ASP Conf. Proc., 315, 92

Staubert, R., Friedrich, S., Pottschmidt, K., Benlloch, S., Schuh, S.L., Kroll, P., Splittgerber, E., \& Rothschild, R. 2003, $A \mathscr{E} A, 407,987$

Szkody P., et al. , 2002, AJ, 123, 413

Tovmassian, G. et al. 2003, PASP, 115, 725

Tovmassian, G.H., Zharikov, S.V., \& Neustroev V. V. 2007, ApJ, 655, 466

Väth, H. 1997, A\&A, 317, 476

Vrielmann, S., Ness, J.-U., \& Schmitt, J.H.M.M. 2005, A\&\&A, 439, 287

Warner, B. 1995, Cataclysmic Variable Stars (CUP: Cambridge)

Warner, B. 2004, PASP, 116, 115

Warner, B., \& Woudt, P.A. 2002, MNRAS, 335, 84

Warner, B., \& Woudt, P.A. 2006, MNRAS, 367, 1562

Warner, B., Woudt, P.A., \& Pretorius, M.L. 2003, MNRAS, 344, 1193

Watson, M.G., King, A.R., \& Osborne, J., 1985, MNRAS, 212, 917

Wheatley, P.J. 1999, in C. Hellier, K. Mukai (eds), Annapolis Workshop on Magnetic Cataclysmic Variables, ASP. Conf. Ser., 157, 47

Wickramasinghe, D.T., \& Ferrario, L. 2000, New Astron. Revs, 44, 69

Williams, G. 2003, PASP, 115, 618

Woudt, P.A., \& Warner, B. 2002, MNRAS, 333, 411

Wynn, G.A., \& King, A.R. 1995, MNRAS, 275, 9

Wynn, G.A., \& King, A.R., 1992, MNRAS, 255, 83

Wynn, G.A., King, A.R., \& Horne, K. 1997, MNRAS, 286, 436 